\title{
Pathology of the dorsal spine in ankylosing hyperostosis
}

\author{
B. VERNON-ROBERTS, C. J. PIRIE, AND V. TRENWITH \\ From the Bone and Joint Research Unit, Institute of Pathology, The London Hospital Medical College
}

While the pathological features of the spine in ankylosing spondylitis have been described in considerable detail in recent years, little published work is available on the subject of the pathology of the spine in ankylosing hyperostosis (AH).

We here describe some of the pathological findings in the dorsal spines of twenty subjects with AH.

\section{Materials and methods}

\section{SELECTION OF SPECIMENS}

Dorsal spines were removed from twenty subjects at postmortem examination. They were judged to have $\mathrm{AH}$ on the basis of the presence of syndesmophytes in the form of bony flanges or bridges situated predominantly on the right anterolateral aspect of the bodies of two or more vertebrae, and the absence of previous clinical findings or other spinal pathology which could account for these features. Radiographs of the dorsal spines taken during life and post-mortem were examined independently by a radiologist and a rheumatologist who confirmed the diagnosis of AH in all the cases included in this study. The twenty cases of AH used in this study were selected from about 500 autopsies where one of us (B.V.-R.) was personally able to examine the spine in each case, and excluded from the study were about ten other cases of $\mathrm{AH}$ where removal of the spine was not possible for a variety of reasons. The overall incidence of $\mathrm{AH}$ in patients coming to autopsy at The London Hospital is therefore in the region of $6 \%$. The twenty subjects comprised eleven males of mean age 66 years (range 58-77 years) and nine females of mean age 71 years (range 53-87 years). Details of the age, sex, extent of the dorsal spine affected, and the principal cause of death in each subject are shown in Table $I$.

\section{EXAMINATION OF SPECIMENS}

After removal from the body, each dorsal spine was cleaned and photographed. Each specimen was then radiographed using a Faxitron 8050-010 $X$-ray Inspection System (Field Emission U.K. Limited). After photography and radiography, 18 of the spines were divided into longitudinal slabs $0.5 \mathrm{~cm}$ thick using a band-saw, and the internal features were noted. Selected portions of these slabs were then taken for histological processing for subsequent microscopical examination. They were fixed in formol-saline and embedded in methyl methacrylate; $8 \mu \mathrm{m}$ sections were made and were stained with haematoxylin and eosin, thionin, and by the von Kossa technique. One severely affected spine and one moderately affected spine were macerated for the examination of the dry specimens by being boiled in $5 \%$ sodium hydroxide to remove the soft tissues, followed by bleaching in $10 \mathrm{vol}$ hydrogen peroxide.

\section{Results}

EXTERNAL FEATURES OF DORSAL SPINE IN AH In 13 of the 20 specimens the condition was confined to the lower half of the dorsal spine; in the remainder, the upper half was also affected (Table I). In all cases at least 4 contiguous vertebral bodies were involved; involvement of the whole of the dorsal spine was present in only one case. The lower part of the affected portion of each spine tended to be more severely affected than the upper, though a region of larger syndesmophytes usually merged with lesser ones above and below.

The varied appearance of the syndesmophytes and their relation to other structures was most easily appreciated by examination of the macerated specimens from which all soft tissues had been removed, since it was only by radiology and palpation through the soft tissues that many of the less marked features could be detected in the intact specimens.

From the macerated specimens it could be seen that the syndesmophytes were predominantly present on the right side of the vertebral bodies: they were also present to a lesser extent on the left side, particularly above the level of the aortic arch and below the level where the aorta leaves the left side of the vertebral bodies to lie on the front of the vertebral column (Fig. 1). The syndesmophytes varied in appearance from relatively small zones of irregular 'lipping' at the margins of the vertebral bodies at their junction with the intervertebral discs, to large bony flanges arising from a broad base incorporating a mass of added bone lying on the front of the right half of each vertebral body (Figs 1, 2, and 3). This mass of added bone lying on the front of the right anterolateral surfaces of the vertebral bodies had a sharply demarcated border on its left side (Fig. 3). Some of the 
Table I Sex, age, cause of death, region of dorsal spine affected, and the presence of bony ankylosis, Schmorl's nodes, and osteoporosis in twenty patients with ankylosing hyperostosis used in the present study

\begin{tabular}{|c|c|c|c|c|c|c|c|}
\hline $\begin{array}{l}\text { Case } \\
\text { no. }\end{array}$ & $\operatorname{Sex}$ & $\begin{array}{l}\text { Age } \\
\text { (yrs) }\end{array}$ & Cause of death & $\begin{array}{l}\text { Dorsal vertebrae } \\
\text { having } \\
\text { syndesmophytes }\end{array}$ & $\begin{array}{l}\text { Bony ankylosis } \\
\text { of } \\
\text { syndesmophytes }\end{array}$ & $\begin{array}{l}\text { Schmorl's } \\
\text { nodes }\end{array}$ & Osteoporosis \\
\hline 1 & $\mathbf{M}$ & 74 & Cerebrovascular accident & $5-11$ & - & + & + \\
\hline 2 & M & 59 & Brain stem tumour & 3-9 & + & + & + \\
\hline$\overline{3}$ & $\mathbf{M}$ & 61 & Hypertension (diabetic) & $6-11$ & - & + & + \\
\hline 4 & $\mathbf{M}$ & 70 & Acute pancreatitis & $4-9$ & - & + & + \\
\hline 5 & $\mathrm{~F}$ & 61 & Biliary peritonitis & $9-10$ & + & - & + \\
\hline 6 & $\mathbf{F}$ & 83 & Fibrosing alveolitis & $5-10$ & + & + & + \\
\hline 7 & $\mathbf{M}$ & 57 & Ischaemic heart disease & $5-8$ & + & + & + \\
\hline 8 & $\mathbf{F}$ & 75 & Ischaemic heart disease & $6-10$ & - & + & + \\
\hline 9 & $\mathbf{F}$ & 57 & Pulmonary embolism & $6-9$ & - & + & + \\
\hline 10 & $\mathbf{M}$ & 61 & Rheumatic heart disease & $6-9$ & - & + & + \\
\hline 11 & $\mathbf{F}$ & 53 & Infective endocarditis & $5-10$ & - & + & + \\
\hline 12 & $\mathbf{M}$ & 72 & Obstructive airways disease & $7-11$ & - & + & + \\
\hline 13 & $\mathbf{F}$ & 63 & Carcinoma of stomach & $6-9$ & - & + & + \\
\hline 14 & M & 77 & Carcinoma of stomach & $6-11$ & - & + & + \\
\hline 15 & $\mathbf{M}$ & 58 & Myelomatosis & $8-11$ & - & - & + \\
\hline 16 & $\mathbf{F}$ & 87 & Cerebrovascular accident & $1-12$ & + & + & + \\
\hline 17 & $\mathbf{M}$ & 61 & Haemorrhage from D.U. & $6-12$ & + & + & + \\
\hline 18 & $\mathbf{F}$ & 76 & Carcinoma of uterus & $7-12$ & + & - & + \\
\hline 19 & $\mathbf{F}$ & 80 & Cerebrovascular accident & $8-12$ & + & + & + \\
\hline 20 & $\mathbf{M}$ & 75 & Cerebrovascular accident & $3-12$ & - & + & + \\
\hline
\end{tabular}

large syndesmophytes from adjoining vertebrae had united by bony ankylosis; but, in some instances, though bony fusion appeared to have taken place radiographically, in fact the adjoining syndesmophytes remained completely separated by a tortuous and narrow cleft occupied by soft tissues having continuity with the underlying disc (Figs 1, 2, and 3).

Examination of the macerated specimens also revealed that small irregular bony outgrowths were present on the dorsal surfaces of the vertebral bodies within the spinal canal, around the articular margins of the apophyseal and costo-vertebral joints (Figs 2 and 3 ), and extending into the supra-spinous ligament from the tips of the spinous processes (Figs 2 and 3 ).

Examination of the fresh nonmacerated specimens showed that the left side of some of the smaller syndesmophytes extended around the right lateral border of the anterior longitudinal ligament to lie in front of this ligament. With syndesmophytes of moderate size, the anterior longitudinal ligament was raised by underlying new bone (Fig. 4) but was not incorporated into the syndesmophytes. Large syndesmophytes displaced the right side of the anterior longitudinal ligament forwards and to the left and gave the appearance of a spiral rotation deformity of the dorsal spine (Fig. 5); though a true rotation deformity of the vertebral bodies was not seen in any specimen.

MICROSCOPICAL FEATURES OF THE DORSAL SPINE IN AH

On the basis of their microscopical appearances, each pair of syndesmophytes from adjoining vertebral bodies was classified into one of the three following grades.

Grade $I=$ small or medium syndesmophytes without bony fusion.

Grade $I I=$ large syndesmophytes without bony fusion.

Grade III = bony ankylosis by fusion of syndesmophytes.

This division is used for descriptive purposes only, and does not imply that all grade I lesions eventually progress to the grade II or III category. However, pairs of syndesmophytes falling into different categories were found in each spine, and a region of large syndesmophytes tended to merge above and below into smaller syndesmophytes.

\section{Grade I syndesmophytes}

A section through the smaller syndesmophytes revealed the presence of an anterolateral extension of fibrous tissue in continuity with the annulus fibrosus on the right side, with syndesmophytes projecting from the margins of the vertebral body above and below to enclose the disc extension (Fig. 6). The sizes of these syndesmophytes enclosing the disc extensions were rarely equal, and sections through medium-sized syndesmophytes enclosing larger disc extensions often revealed that the lateral extension from the annulus fibrosus had been largely surrounded by the syndesmophyte from the vertebra below alone (Fig. 7); and it is easy to see why this latter type of syndesmophyte gives rise to the 'candle-flame' appearance 

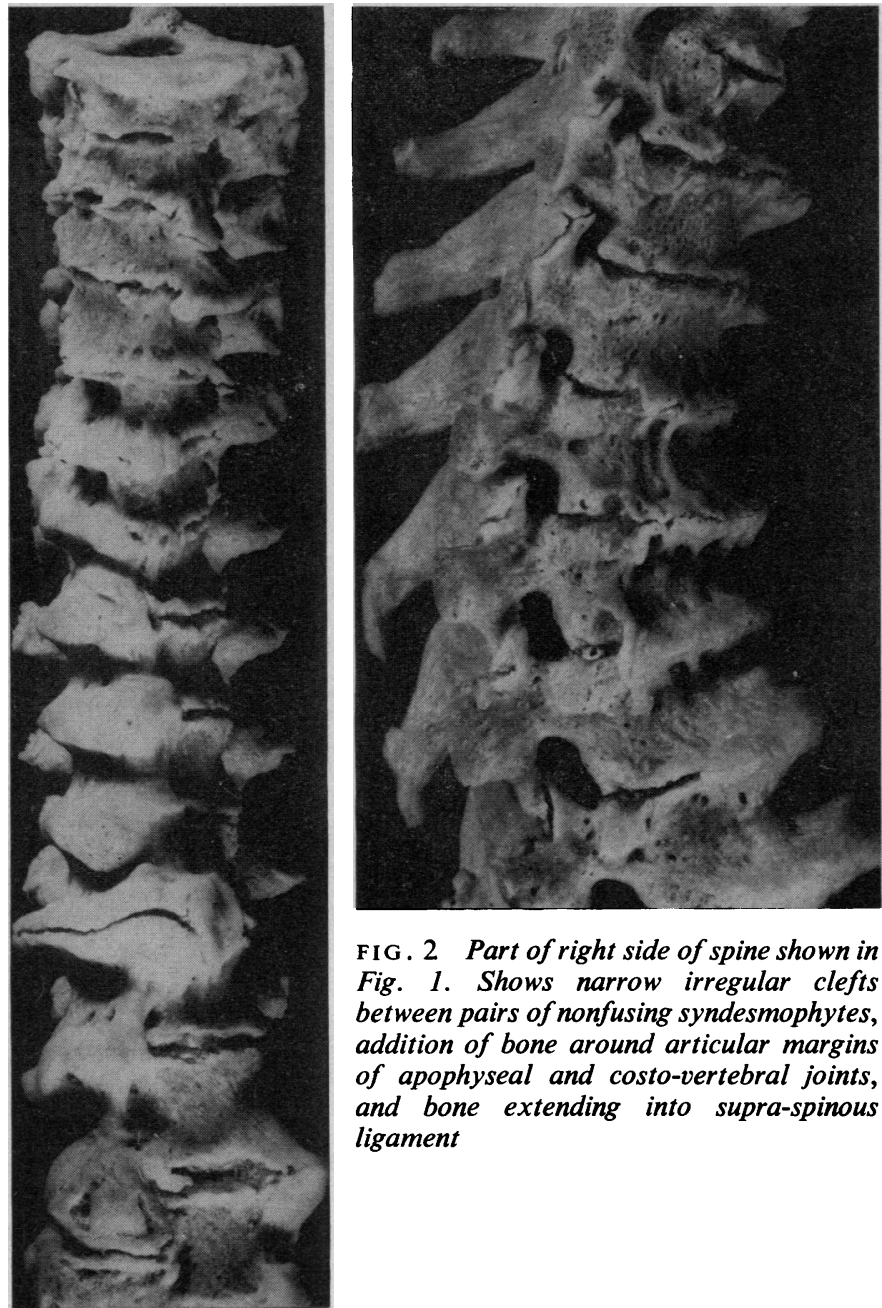

FIG. 2 Part of right side of spine shown in Fig. 1. Shows narrow irregular clefts between pairs of nonfusing syndesmophytes, addition of bone around articular margins of apophyseal and costo-vertebral joints, and bone extending into supra-spinous ligament

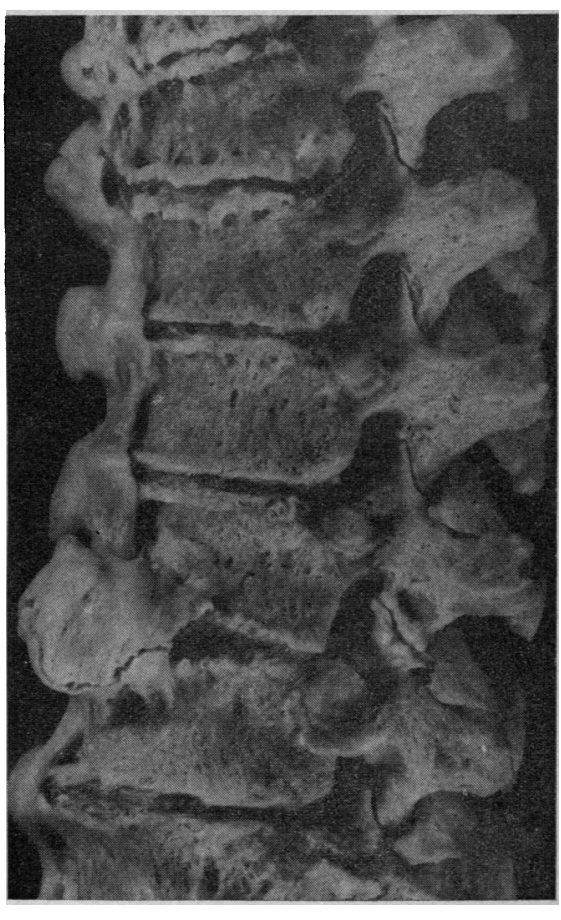

FIG. 3 Part of left side of spine shown in Fig. 1. The bone added to the right side of each vertebral body has a distinct left margin with, in places, an underlying space where it has extended over the anterior longitudinal ligament. Note also the addition of bone around the articular margins of the apophyseal and costo-vertebral joints
FIG. 1 Anterior view of macerated dorsal spine. Shows broad-based large syndesmophytes, some of which have fused, situated on the right side below the level of $D 4$ and on the left side above D4 and below D10. Note narrow clefts between nonfused pairs of syndesmophytes, and bone which has been added to the right anterolateral surfaces of the vertebral bodies

on $x$-ray, though the sectioned syndesmophyte is part of a broad-based flange of bone and is not a discrete spike as the $x$-ray may suggest.

Marked osteoporosis, evidenced by widespread and uniform loss of cancellous bone from vertebral bodies, was present in the majority of specimens examined (Figs 6 and 7). However, associated with medium-sized syndesmophytes was the presence of moderately dense bone thickening the bone plate underlying the hyaline lamina and the tissue extending from the annulus fibrosus (Fig. 7).
Vascular invasion of the lateral edge of the annulus fibrosus (Fig. 8) was occasionally seen and was associated with the presence of small syndesmophytes. Separate discrete 'islands' of bone were also sometimes present within the lateral extension from the annulus or adjoining syndesmophytes (Fig. 7).

Frequently present were Schmorl's nodes formed by herniation of part of the nucleus pulposus into the cancellous bone of the vertebral body through a defect in the hyaline lamina and subchondral bone plate (Fig. 6). Despite this loss of nuclear material and the lateral extensions from the annulus, the disc thickness was frequently normal with no evidence suggesting focal or diffuse narrowing. In some cases, the extruded nuclear material of the Schmorl's node was largely replaced by vascular granulation tissue which also extended through the gap in the bone plate into the remaining nucleus pulposus (Fig. 9). 


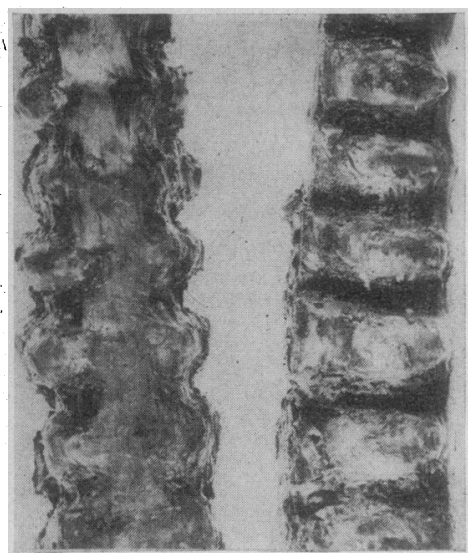

FIG. 4 Anterior view (left) and right side (right) of freshly removed dorsal spine with neural arches removed. Shows moderately large syndesmophytes raising the anterior longitudinal ligament

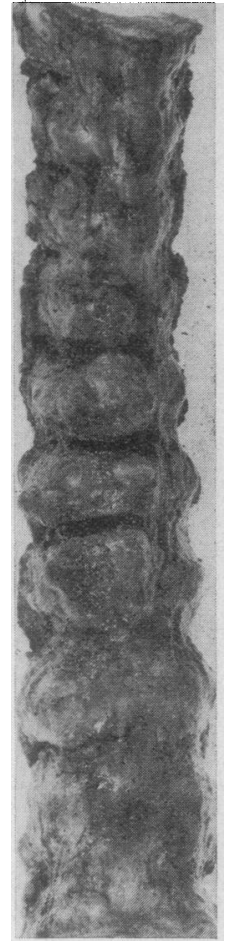

FIG. 5 Anterior view of freshly removed dorsal spine. The very large right-sided syndesmophytes in the mid-portion of the specimen have deviated the anterior longitudinal ligament to the left

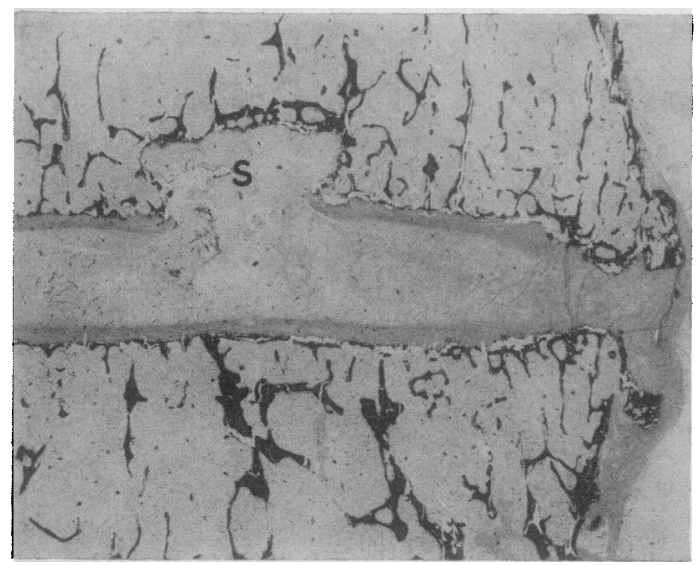

FIG. 6 Section through small grade I syndesmophyte enclosing fibrous tissue continuous with the annulus fibrosus. Despite the presence of a Schmorl's node $(S)$, the disc space is normal. There is marked osteoporosis. von Kossa. $\times 4$

Grade II syndesmophytes

Sections through adjoining pairs of large nonankylosed syndesmophytes revealed large intervening extensions of fibrous tissue retaining continuity with the annulus fibrosus, and these extensions were fre-

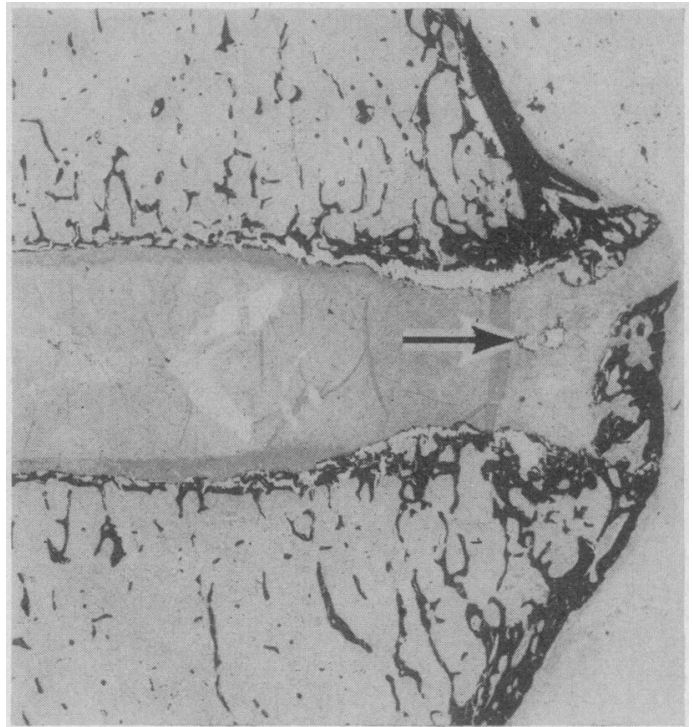

FIG. 7 Section through moderate-sized grade I syndesmophyte. The lateral extension of fibrous tissue from the annulus is largely enclosed by the syndesmophyte arising from the lower vertebral body. Dense bone forms the syndesmophyte, and forms a thin layer underlying the lateral part of the disc and on the surface of the vertebral body. There is marked osteoporosis in the vertebral body. The disc width is normal, and a small 'island' of bone (arrow) is present. von Kossa. $\times 5$

quently T-shaped or Y-shaped (Figs $10 a$ and $b$ ). At their outer margins the syndesmophytes were narrowly separated by fibrous tissue. The tortuous separation of the closely-approximated syndesmophytes explained why it was frequently and erroneously concluded that bony ankylosis had taken place after examination of radiographs of the dorsal spine. In some instances, the fibrous tissue extending from the annulus was largely surrounded by a syndesmophyte arising from the lower vertebral body alone (Figs $10 a$ and $b$ ). Occasionally, syndesmophytes extended into the deep surface of the anterior longitudinal ligament by the endochondral ossification of metaplastic cartilage (Fig. 11), but there was no evidence that this process contributed significantly to the size of syndesmophytes or to the accretion of bone on the fronts of vertebral bodies.

Much dense bone had formed on the front of each vertebral body, and also thickened the bone plate underlying the lateral extension from the annulus and adjacent hyaline lamina (Fig. 10b). Elsewhere, the vertebral bodies exhibited marked osteoporosis (Figs $10 a$ and $b$ ).

Despite the frequency of Schmorl's nodes, defects of the hyaline lamina, and irregularities of the bone plate, the disc thickness was not greatly reduced in the majority of cases. However, some degree of general narrowing of the disc was observed in some instances (Fig. 10b). 


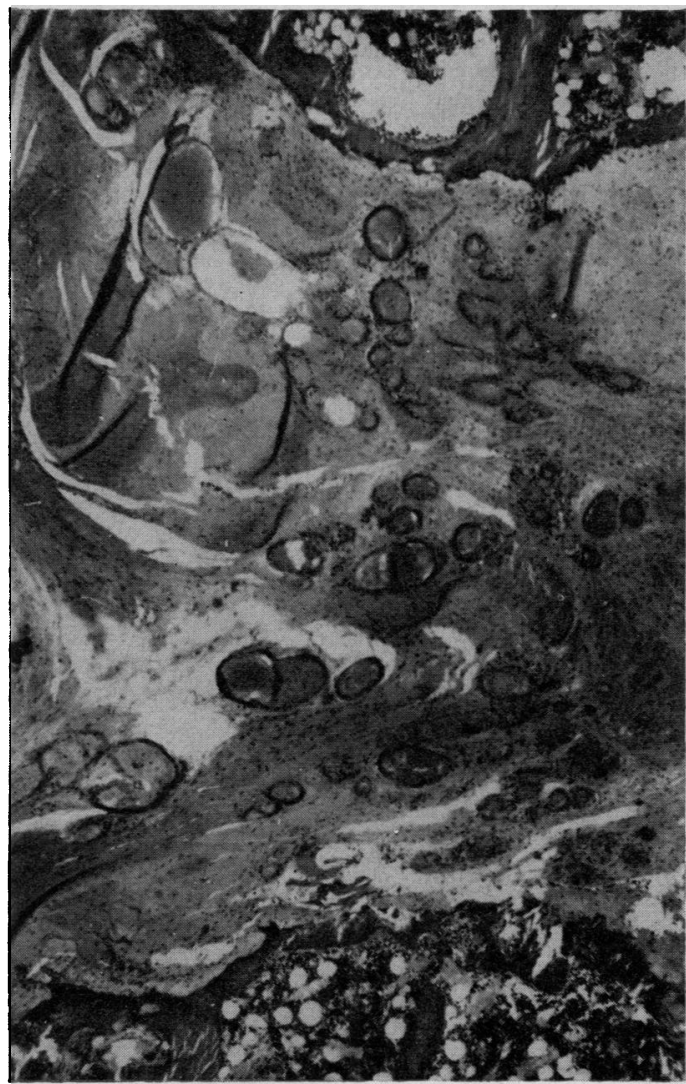

FIG. 8 Numerous blood vessels lying within the edge of the annulus fibrosus in the area between small syndesmophytes. Haematoxylin and eosin. $\times 100$

\section{Grade III syndesmophytes}

Sections through adjoining pairs of syndesmophytes which had fused by bony ankylosis revealed the dense bone which formed the syndesmophytes, thickened the anterolateral region of the vertebral bodies, and increased the thickness of the bone plate underlying the lateral extension from the annulus and the adjacent hyaline lamina (Fig. 12). The extensions of fibrous tissue from the annulus were also largely replaced by dense bone (Fig. 12). By contrast, there was marked osteoporosis of the remainder of the vertebral bodies in the majority of cases (Fig. 12).

While the bone plate on each of the intervertebral disc was frequently irregular and Schmorl's nodes were often present, definite narrowing of the disc was uncommon; but, in one spine, the discs between D7, 8 , and 9 had almost disappeared and bony fusion had taken place between the vertebral bodies across the discs (Fig. 13).

\section{Discussion}

The pathological features observed, and their frequency in the 20 spines examined in this study, are summarized in Table II.

It is clearly a matter of conjecture to postulate a dynamic sequence of events from the examination of postmortem material, particularly in view of the fact that varying grades of 'severity' of the hyperostotic syndesmophytes were always seen in the same spine. Moreover, the varying incidence of bony ankylosis in relation to the age of the subjects in this study indicates that there are individual variations in the rate at which the condition progresses and the ultimate severity achieved. However, taking into account the fact that a region of large syndesmophytes usually

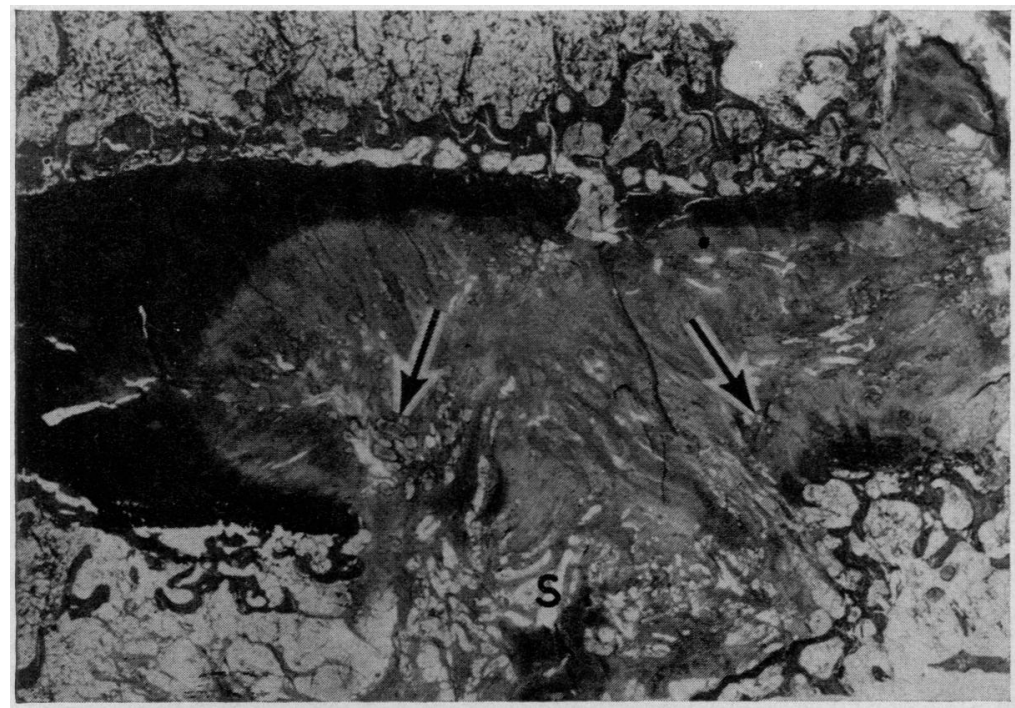

FIG.9 Section throughorganizing Schmorl's node $(S)$ showing vascular granulation tissue (arrows) extending into the disc through the gap in the bone plate and hyaline lamina. Haematoxylin and eosin. $\times 50$ 


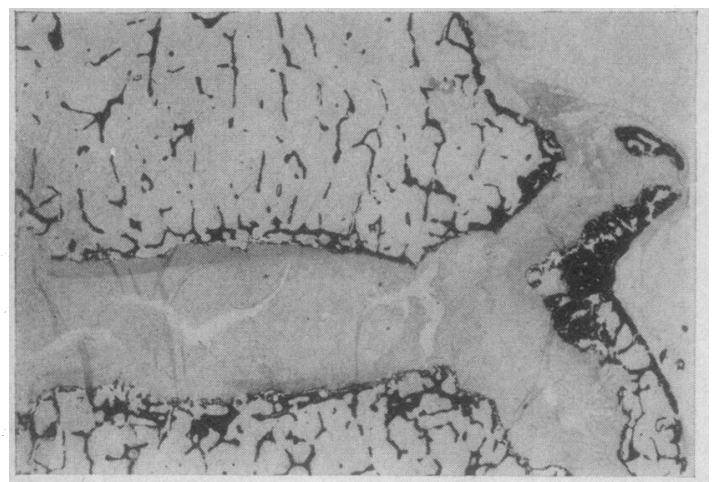

(a)

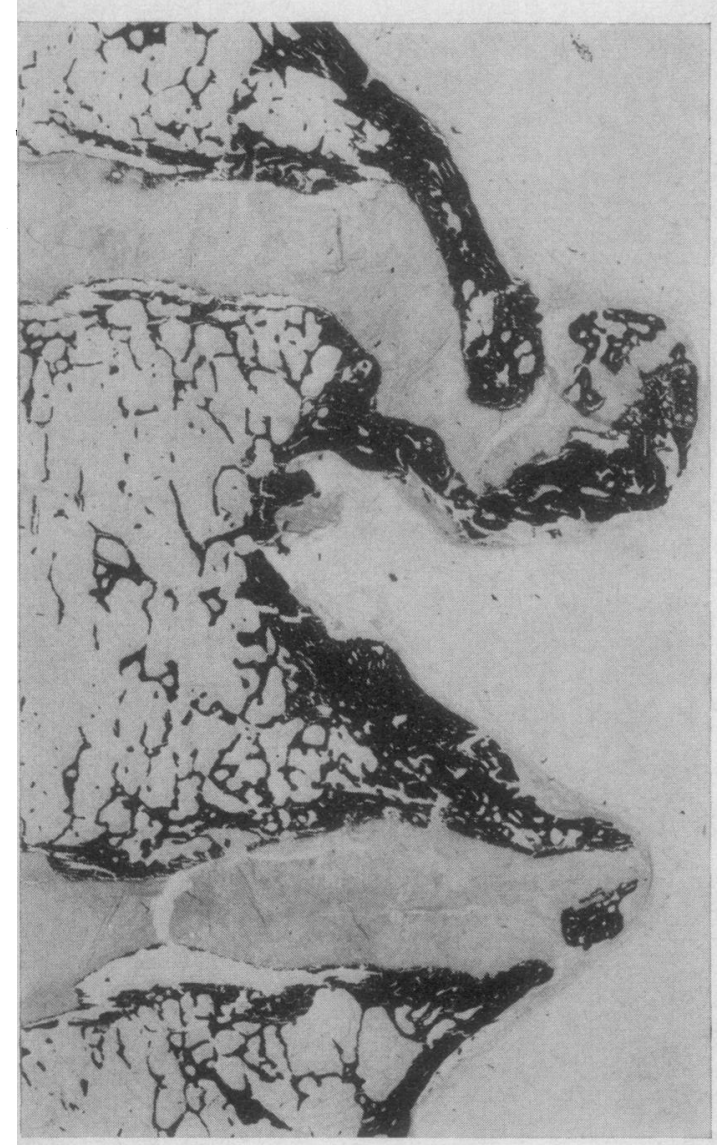

(b)

FIG. 10 Sections through grade II syndesmophytes. The syndesmophytes are narrowly separated by fibrous tissue continuous with the annulus. The fibrous tissue extending from the annulus may be T-shaped or Y-shaped (10a) and may be largely enclosed by the syndesmophyte arising from the lower vertebral body (10b). Dense bone has formed around the syndesmophytes and on the surface of the vertebral bodies, but there is marked osteoporosis elsewhere. The disc width is well maintained. von Kossa. $\times 5$

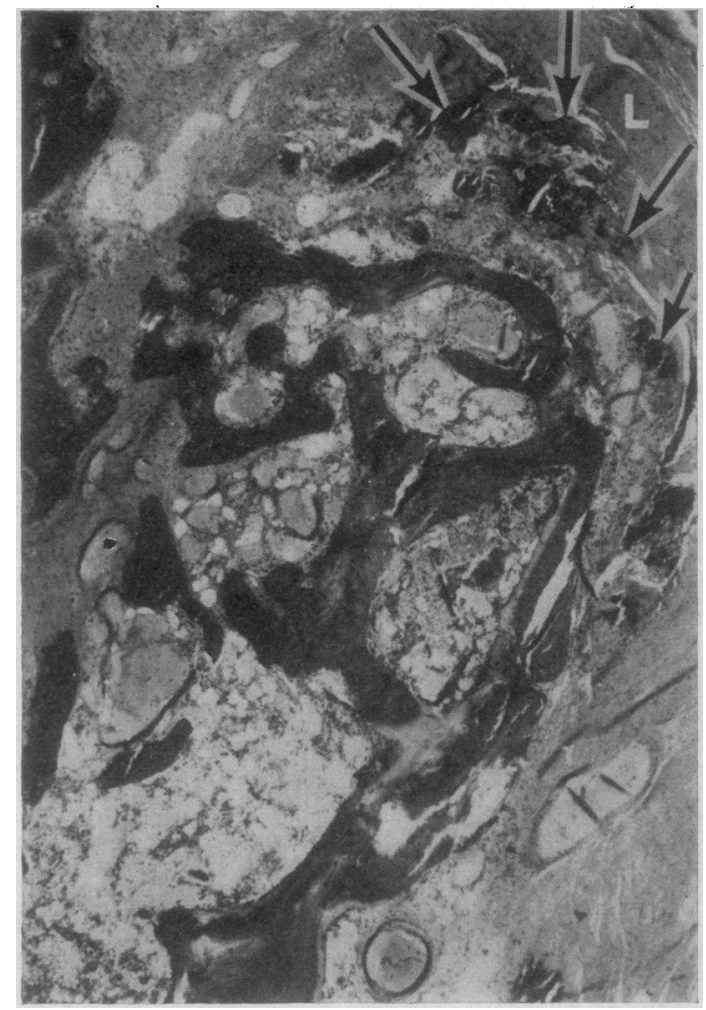

FIG. 11 Section through grade II syndesmophyte which has extended into the deep surface of the anterior longitudinal ligament $(L)$ by progressive ossification of metaplastic cartilage (arrows). Haematoxylin and eosin. $\times 75$

Table II Frequency of pathological findings in the dorsal spines of twenty patients with ankylosing hyperostosis

\begin{tabular}{|c|c|c|}
\hline \multirow[b]{2}{*}{ Pathological feature } & \multicolumn{2}{|c|}{ Cases present } \\
\hline & No. & Per cent. \\
\hline $\begin{array}{l}\text { Syndesmophytes } \\
\text { Fibrous tissue extending laterally }\end{array}$ & $\begin{array}{l}20 \\
20\end{array}$ & $\begin{array}{l}100 \\
100\end{array}$ \\
\hline $\begin{array}{l}\text { Accretion of bone on the surfaces } \\
\text { of vertebral bodies }\end{array}$ & 20 & 100 \\
\hline $\begin{array}{l}\text { Osteoporosis } \\
\text { Schmorl's nodes } \\
\text { Bony ankylosis of syndesmophytes } \\
\text { Islands of bone adjacent to } \\
\text { syndesmophytes }\end{array}$ & $\begin{array}{r}20 \\
17 \\
8 \\
4\end{array}$ & $\begin{array}{r}100 \\
85 \\
40 \\
20\end{array}$ \\
\hline $\begin{array}{l}\text { Ossification of anterior longitudinal } \\
\text { ligament }\end{array}$ & 3 & 15 \\
\hline $\begin{array}{l}\text { Marked narrowing of some } \\
\text { intervertebral discs }\end{array}$ & 2 & 10 \\
\hline $\begin{array}{l}\text { All intervertebral discs narrowed and } \\
\text { some partly obliterated by bone }\end{array}$ & 1 & 5 \\
\hline
\end{tabular}




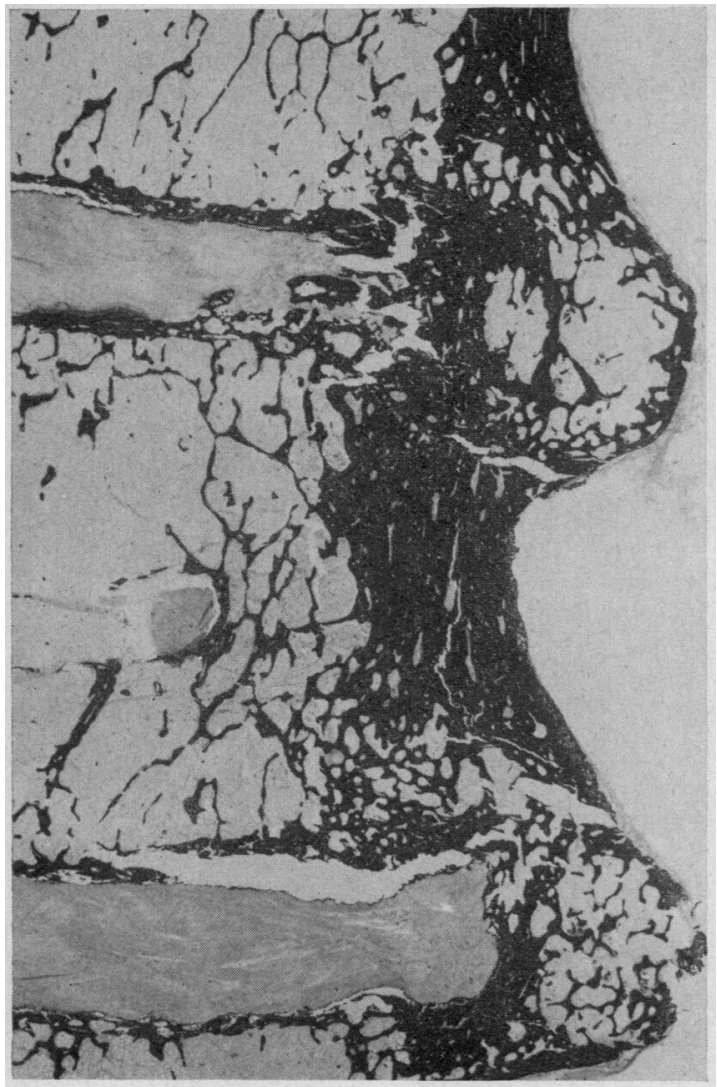

FIG.12 Section through grade III syndesmophyte showing bony fusion. There has been heavy accretion of dense bone on the surface of the vertebral body and in the bone plate region, but there is marked osteoporosis elsewhere. The disc width is well maintained. von Kossa. $\times 5$

merges cranially and caudally into progressively smaller syndesmophytes, the histological findings suggest that the initial event in AH may be a predominantly right-sided anterolateral extension of fibrous tissue in continuity with the annulus fibrosus of the intervertebral disc. This process may be inhibited on the left side by the presence of the descending thoracic aorta. The T-shaped or Y-shaped nature of this fibrous tissue extending from the annulus in larger syndesmophytes suggests that the tissue extends upwards and downwards due to the presence of periosteum covering the external surface of each vertebral body. Since elevation of periosteum leads to underlying new bone formation, this could explain the particular flange-shaped nature (and 'candleflame' radiological appearance) of the syndesmophytes in $\mathrm{AH}$, and also the accretion of new bone on the right anterolateral surface of each vertebral body. With the eventual close proximity of the larger syndesmophytes, it is not surprising that bony ankylosis occurs in some instances; however, bony ankylosis

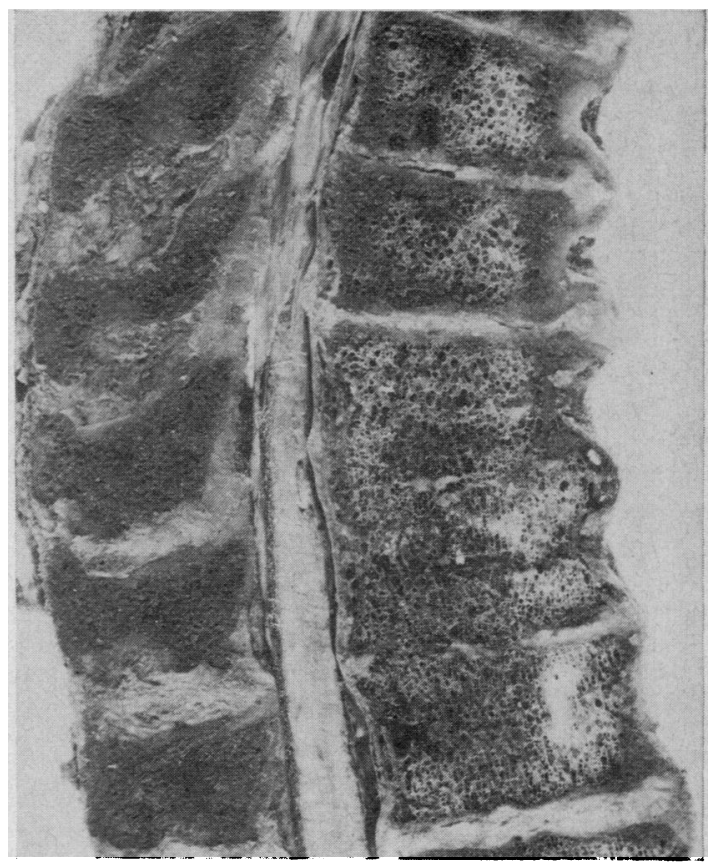

FIG. 13 View of sagittal section through freshly removed dorsal spine. Shows marked narrowing of discs, with discs between D7, 8, and 9 largely obliterated by bony fusion between the vertebral bodies. These appearances seen in this spine only.

in $\mathrm{AH}$ appears to be much less frequent than clinical radiographs suggest.

Unlike the pathogenesis of spinal osteophytosis ('spondylosis deformans') proposed by Collins (1949), the above postulated mechanism for syndesmophyte formation in $\mathrm{AH}$ does not implicate disc prolapse or protrusion as an initial event, since despite the frequent presence of Schmorl's nodes in AH they were not present in all cases. Moreover, normal disc thickness was often maintained in AH despite the presence of Schmorl's nodes and large syndesmophytes, and the incidence of Schmorl's nodes at autopsy (Schmorl and Junghanns, 1932) is much higher $(38 \%)$ than the incidence of $\mathrm{AH}(6 \%)$ in this study. The preservation of disc thickness could be related to the osteoporosis which we have found as a constant and marked feature, since our studies of the lumbar spine reveal that severe osteoporosis tends to protect the discs from compression and degeneration (B. Vernon-Roberts and C. J. Pirie, unpublished observations). It thus seems unlikely that some inherited, congenital, or acquired defect in the hyaline lamina or bony end plate of the vertebral body plays a part in initiating the changes which result in the clinical and pathological appearance of $\mathrm{AH}$.

We have observed an increase in the vascularity of the edge of the annulus fibrosus in association with 
small syndesmophytes in some cases of $\mathrm{AH}$, and this could be associated with the need for an increased blood supply for the formation of new fibrous tissues and bone.

Unlike Forestier and Lagier (1971) we have not found that extensive ossification of the anterior longitudinal ligament is a constant or marked feature of AH. However, in agreement with others (Forestier and Lagier, 1971 ; Bywaters, 1972) we have occasionally observed syndesmophytes extending into the deep surface of the anterior longitudinal ligament, but we believe that this process does not contribute significantly to the extensive accretion of new bone on the anterior surface of the vertebral bodies seen in advanced $\mathrm{AH}$.

The findings by our clinical colleagues of hyperostosis occurring in parts of the skeleton other than the spine (Harris, Carter, Glick, and Storey, 1974) indicate that the spinal manifestations of AH are only part of some generalized congenital or acquired abnormality of the skeletal tissues. Despite the formation of ankylosing syndesmophytes in both conditions, there is no clinical or histopathological basis for postulating a common pathogenesis for $\mathrm{AH}$ and ankylosing spondylitis. However, it is of interest that the spine in $\mathrm{AH}$ has radiological and pathological similarities to the spine in acromegaly (Bluestone, Bywaters, Hartog, Holt, and Hyde, 1971). Thus, in acromegaly there is syndesmophytosis which sometimes extends to bony bridging, accretion of bone which increases the width of vertebral bodies, and an anterolateral extension of disc tissues with the retention or increase of disc thickness and the absence of disc degeneration (Bluestone and others, 1971; Jaffe, 1972). By contrast, there are features of acromegaly, in the spine and other parts of the body, which are not present in AH. Moreover, growth hormone levels are not raised in patients having AH (Harris and others, 1974); and we have not found evidence supporting an association of AH with diabetes mellitus, since the latter condition was present in only one of the twenty patients in our study and in four of the 34 patients studied by Harris and others (1974). On the basis of present knowledge, there is therefore no strong evidence to support the postulate that $\mathrm{AH}$ may be a manifestation of acromegaly or of the unbalanced or unopposed action of growth hormone in 'susceptible' individuals. We conclude that, until evidence to the contrary is adduced, AH should be regarded as a condition of unknown pathogenesis, and quite distinct from acromegaly or other form of skeletal disease.

\section{Summary}

The macroscopical and microscopical pathological findings in dorsal spines from twenty patients with ankylosing hyperostosis are described.

(1) Pairs of syndesmophytes arising from the anterolateral margins of adjoining vertebral bodies were separated by intervening fibrous tissue in continuity with the annulus fibrosus of the intervertebral disc. (2) Normal disc thickness was usually retained despite syndesmophyte formation and the frequent presence of Schmorl's nodes.

(3) The close proximity and irregular adjoining surfaces of pairs of non-ankylosed syndesmophytes often gave rise to the radiological appearance of bony fusion, but true bony ankylosis was present in only 8 of the 20 spines examined.

(4) The heavy accretion of bone on the right anterolateral surfaces of the vertebral bodies was not due to ossification of the anterior longitudinal ligament, although there was infrequent and limited extension of some syndesmophytes into the ligament by a process of endochondral ossification of metaplastic cartilage,

(5) There was widespread osteoporosis in the vertebral bodies.

(6) Osteophytic outgrowths of bone were present around the margins of the apophyseal and costovertebral joints, and bone extended into the supraspinous ligament.

(7) The pathological findings do not favour a pathogenetic relationship between ankylosing hyperostosis and ankylosing spondylitis, acromegaly, or other forms of skeletal disease.

We record our gratitude to Dr. J. Harris and Dr. A. R. Carter for examining radiographs, and thank the Arthritis and Rheumatism Council, the Medical Research Council, and The London Hospital Medical College for their generous support.

\section{References}

Bluestone, R., Bywaters, E. G. L., Hartog, M., Holt, P. J. L., and Hyde, S. (1971) Ann. rheum. Dis., 30, 243 (Acromegalic arthropathy)

Bywaters, E. G. L. (1972) Discussion of the pathology of ankylosing hyperostosis at meeting of Society for

Back Pain Research at The Royal College of Surgeons in November 1972

Collins, D. H. (1949) 'The Pathology of Articular and Spinal Disease'. Arnold, London

Forestier, J., AND LAGIER, R. (1971) Clin. Orthop., 74, 65 (Ankylosing hyperostosis of the spine)

Harris, J., Carter, A. R., Glick, E. N., And Storey, G. O. (1974) Ann. rheum. Dis., 33, 210

(Ankylosing hyperostosis. Clinical and radiological features)

JAFFE, H. L. (1972) 'Metabolic, Degenerative, and Inflammatory Diseases of Bones and Joints'. Lea and Febiger, Philadelphia

SchmoRL, G., AND JunghanNs, H. (1932) 'Die gesund und kranke Wirbelsäule im Röntgenbild'. Georg Thieme, Leipzig 